\title{
Comparison between different cone-beam computed tomography devices in the detection of mechanically simulated peri-implant bone defects
}

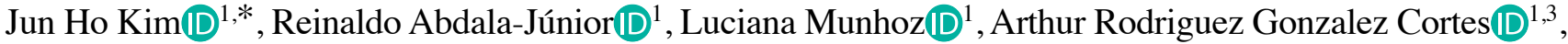 \\ Plauto Christopher Aranha Watanabe $\mathbb{D}^{2}$, Claudio Costa $\mathbb{D}^{1}$, Emiko Saito Arita $\mathbb{D}^{1}$ \\ ${ }^{1}$ Department of Stomatology, School of Dentistry, University of São Paulo, São Paulo, Brazil \\ ${ }^{2}$ Department of Stomatology, Field of Public Oral Health and Forensic Dentistry, School of Dentistry of Ribeirão Preto, University of \\ São Paulo, Ribeirao Preto, Brazil \\ ${ }^{3}$ Department of Dental Surgery, Faculty of Dental Surgery, University of Malta, Mater Dei Hospital, Msida, Malta
}

\section{ABSTRACT}

Purpose: This study compared 2 cone-beam computed tomography (CBCT) systems in the detection of mechanically simulated peri-implant buccal bone defects in dry human mandibles.

Materials and Methods: Twenty-four implants were placed in 7 dry human mandibles. Peri-implant bone defects were created in the buccal plates of 16 implants using spherical burs. All mandibles were scanned using 2 CBCT systems with their commonly used acquisition protocols: i-CAT Gendex CB-500 (Imaging Sciences, Hatfield, PA, USA; field of view [FOV], $8 \mathrm{~cm} \times 8 \mathrm{~cm}$; voxel size, $0.125 \mathrm{~mm} ; 120 \mathrm{kVp} ; 5 \mathrm{~mA} ; 23 \mathrm{~s}$ ) and Orthopantomograph OP300 (Intrumentarium, Tuusula, Finland; FOV, $6 \mathrm{~cm} \times 8 \mathrm{~cm}$; voxel size, $0.085 \mathrm{~mm} ; 90 \mathrm{kVp} ; 6.3 \mathrm{~mA} ; 13 \mathrm{~s}$ ). Two oral and maxillofacial radiologists assessed the CBCT images for the presence of a defect and measured the depth of the bone defects. Diagnostic performance was compared in terms of the area under the curve (AUC), accuracy, sensitivity, specificity, and intraclass correlation coefficient.

Results: High intraobserver and interobserver agreement was found $(P<0.05)$. The OP300 showed slightly better diagnostic performance and higher detection rates than the CB-500 (AUC, $0.56 \pm 0.03$ ), with a mean accuracy of $75.0 \%$, sensitivity of $81.2 \%$, and specificity of $62.5 \%$. Higher contrast was observed with the CB-500, whereas the OP300 formed more artifacts.

Conclusion: Within the limitations of this study, the present results suggest that the choice of CBCT systems with their respective commonly used acquisition protocols does not significantly affect diagnostic performance in detecting and measuring buccal peri-implant bone loss.(Imaging Sci Dent 2020; 50: 133-9)

KEY WORDS: Peri-Implantitis; Dental Implants; Cone-Beam Computed Tomography

\section{Introduction}

Peri-implantitis is characterized by an inflammatory process around an implant, which ranges from soft tissue inflammation to progressive bone loss. ${ }^{1}$ Image overlapping of adjacent anatomical structures on intraoral

We wish to thank CAPES (Coordination of the Advancement of Higher Education, Brasília, Brazil) for providing a MS grant (J.H.K.).

Received September 7, 2019; Revised April 12, 2020; Accepted April 24, 2020

*Correspondence to : Dr. Jun Ho Kim

Department of Stomatology, School of Dentistry, University of São Paulo, Av. Professor Lineu Prestes, $n^{\circ}$ 2227. Zip code: 05508-000. São Paulo, SP, Brazil Tel) 55-11-3091-7831,E-mail) jun.kim@usp.br radiographs may prevent the detection of buccal and lingual peri-implant bone defects. ${ }^{2,3}$ In contrast, cone-beam computed tomography (CBCT) provides cross-sectional images of buccal and lingual plates. ${ }^{4-10} \mathrm{CBCT}$ images have been described as more effective for the detection of peri-implant bone defects than conventional radiographic methods. ${ }^{4,5,9}$

Despite the usefulness of CBCT in the detection of periimplant bone defects, ${ }^{4,5,7,10,11}$ beam-hardening artifacts from dental implants may affect its diagnostic performance. ${ }^{12,13}$ In this context, 3 previous studies reported that CBCT was less accurate for assessing peri-implant bone loss than in- 
traoral radiography. ${ }^{14-16}$ Metal artifact reduction algorithms were tested to improve the detection of peri-implant defects on CBCT images, and also showed unclear results.

Several authors have tested whether different acquisition parameters on CBCT devices reduce artifact formation and improve the peri-implant defect detection rate..$^{4,5,711} \mathrm{Al}$ though the effects of field-of-view (FOV) size and number of frames have been well documented, ${ }^{4,7}$ the influence of other acquisition parameters such as kilovoltage peak $(\mathrm{kVp})$ values in several acquisition protocols commonly used for dental implants remains unclear. To our knowledge, only 1 recent study has investigated the effectiveness of CBCT images obtained with different $\mathrm{kVp}$ settings in the detection of peri-implant defects. The aforementioned authors suggested that higher $\mathrm{kVp}$ values led to a higher detection rate of bone defects. ${ }^{11}$

Thus, the aim of this study was to compare 2 different CBCT devices with their commonly used acquisition protocols for dental implants in the detection of mechanically simulated peri-implant buccal bone defects in dry human mandibles.

\section{Materials and Methods}

The present study was approved by the Ethics Committee of the School of Dentistry of the University of São Paulo, under number CAAE 67441817.7.0000.0075.

An oral surgeon placed 24 cylindrical titanium dental implants to the alveolar bone crest level in 7 human dry edentulous mandibles. In order to avoid the influence of beam-hardening artifacts, a maximum of 4 implants with a minimum inter-implant distance of $20 \mathrm{~mm}$ were placed per mandible. All implants were placed in posterior mandibular sites (i.e., either first lower premolar or second lower molar sites). After implant placement, buccal bone defects were created with a spherical bur $(3017 \mathrm{HL}$; KG Sorensen, São Paulo, Brazil). Peri-circumferential defects with a diameter of $2.0 \mathrm{~mm}$ were created at 16 random implant sites, whereas the other 8 implant sites remained with no defects. All the defect depths were measured by a periodontal probe (North Carolina \#15, Hu-Friedy, Chicago, IL, USA), and confirmed with a digital caliper. The depths were $0.5 \mathrm{~mm}, 1.0 \mathrm{~mm}, 2.5 \mathrm{~mm}, 3.0 \mathrm{~mm}, 3.5 \mathrm{~mm}$, $4.5 \mathrm{~mm}, 7.0 \mathrm{~mm}$, and $15.0 \mathrm{~mm}$, simulating differences in the severity of peri-implantitis, as shown in Figure 1.

\section{CBCT imaging}

Red wax was applied to the mandibles to simulate softtissue attenuation, as described previously. ${ }^{5}$ Two CBCT

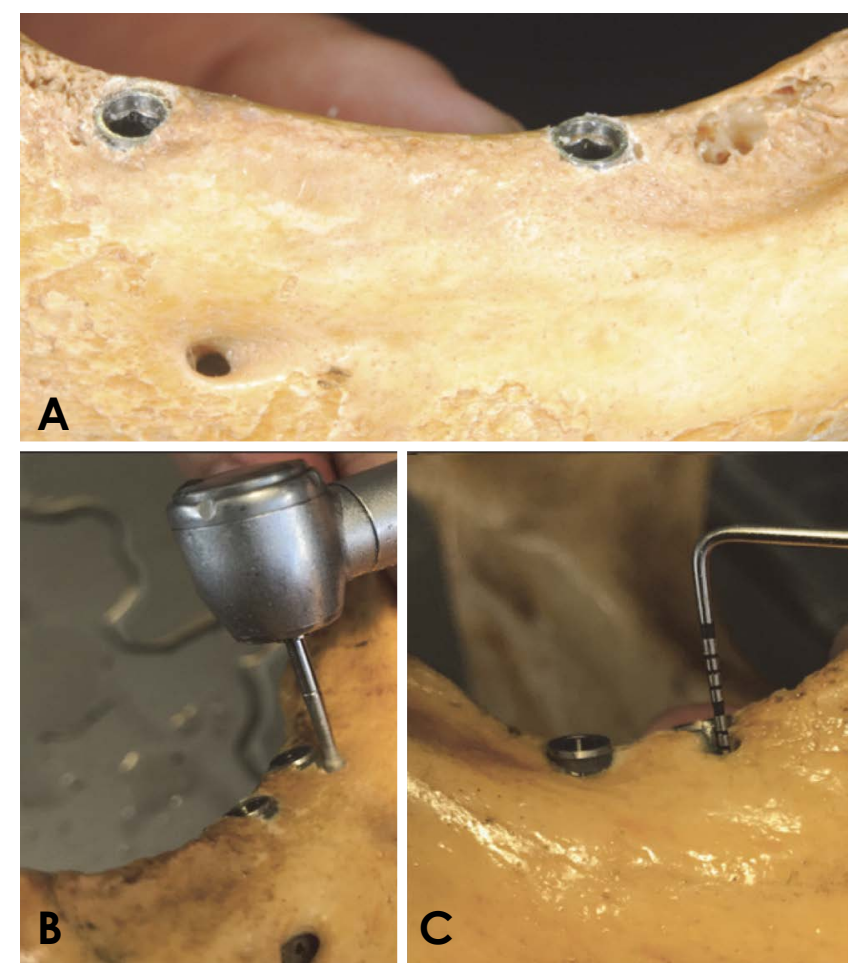

Fig. 1. A. Implants placed to the alveolar bone crest level in the mandible. B. After implant placement, buccal bone defects are created with a spherical bur. C. The defect depths are measured by a periodontal probe.

devices were used: i-CAT Gendex CB-500 (Imaging Sciences, Hatfield, PA, USA; FOV, $8 \mathrm{~cm} \times 8 \mathrm{~cm}$; voxel size, $0.125 \mathrm{~mm} ; 120 \mathrm{kVp} ; 5 \mathrm{~mA} ; 23 \mathrm{~s})$ and Orthopantomograph OP300 (Intrumentarium, Tuusula, Finland, FOV, $6 \mathrm{~cm} \times 8$ $\mathrm{cm}$; voxel size, $0.085 \mathrm{~mm}$; $90 \mathrm{kVp} ; 6.3 \mathrm{~mA} ; 13 \mathrm{~s}$ ). The aforementioned acquisition protocols are among the most commonly used in implant dentistry for these 2 respective CBCT devices.

The CBCT images were obtained by an oral and maxillofacial radiologist as DICOM files, and then exported as TIFF files using imaging software (Adobe Photoshop CS 8.0, Adobe Systems Inc., San Jose, CA, USA).

\section{Data collection and image analysis}

Two oral and maxillofacial radiologists participated in this study as observers. A total of 48 TIFF images of crosssectional CBCT slices were selected and analyzed. The diagnostic performance of the 2 devices was also compared. Before image analysis, both observers were calibrated using representative pilot images. The differences between bone defects and artifacts were explained to both observers using presentation software (Microsoft PowerPoint for Mac; Microsoft Corporation, Redmond, WA, USA). The 


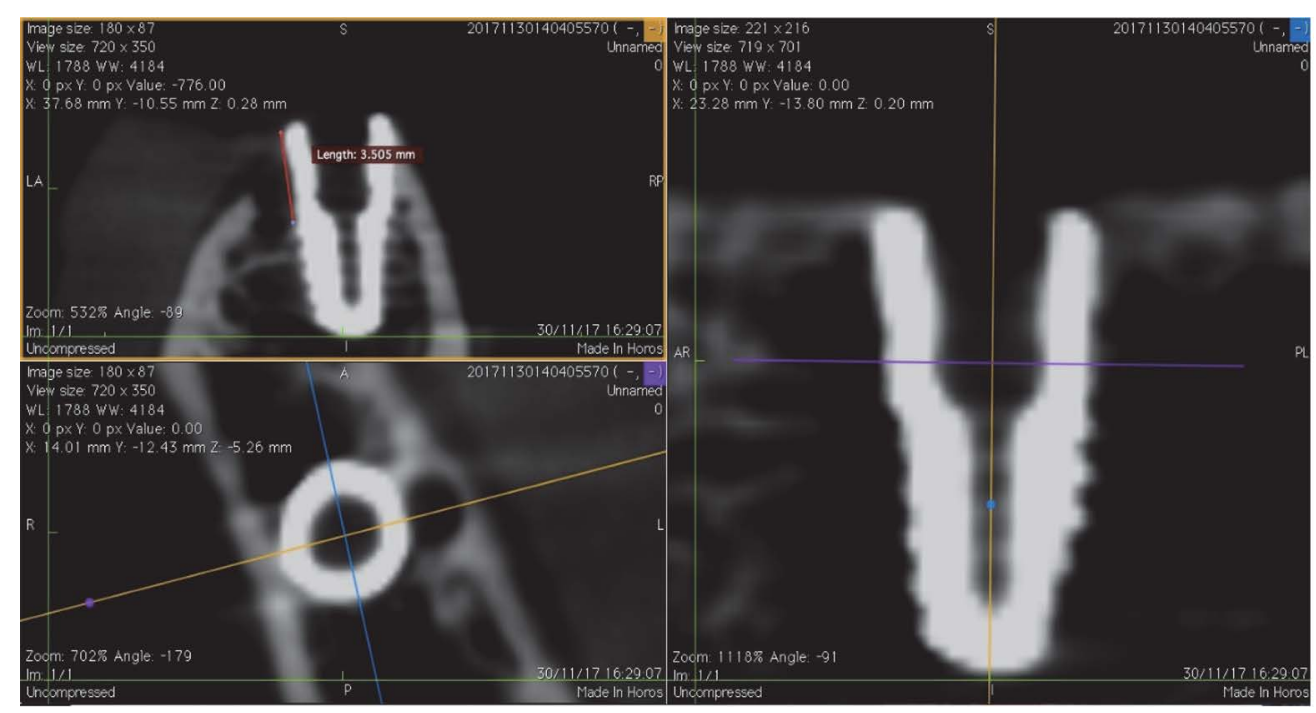

Fig. 2. Quantitative defect depths are measured on software.

Table 1. Intraobserver and interobserver agreement (kappa) for the qualitative assessment of peri-implant defects

\begin{tabular}{|c|c|c|c|c|}
\hline & \multicolumn{2}{|c|}{ i-CAT Gendex CB-500 (120kVp) } & \multicolumn{2}{|c|}{ Orthopantomograph OP300 (90kVp) } \\
\hline & Observer 1 & Observer 2 & Observer 1 & Observer 2 \\
\hline Observer 1 & $0.87 *$ & $0.61 *$ & $0.91 *$ & $0.65^{*}$ \\
\hline Observer 2 & & $0.86^{*}$ & & $0.88 *$ \\
\hline
\end{tabular}

$* P<0.05$

Table 2. Intra- and interobserver agreement (intraclass correlation coefficients) for the quantitative assessment of peri-implant defects

\begin{tabular}{ccccc}
\hline & \multicolumn{2}{c}{ i-CAT Gendex CB-500 $(120 \mathrm{kVp})$} & \multicolumn{2}{c}{ Orthopantomograph OP300 (90kVp) } \\
& Observer 1 & Observer 2 & Observer 1 & Observer 2 \\
\hline Observer 1 & $0.93^{*}$ & $0.92^{*}$ & $0.83^{*}$ & $0.70^{*}$ \\
Observer 2 & & $0.95^{*}$ & & $0.89 *$ \\
\hline
\end{tabular}

$* P<0.05$

images used in this training exercise were discarded before initiating the image assessment of the present study.

Both observers were asked to make qualitative assessments by rating the presence or absence of a bone defect according to a 5-point scale; 1: definitely absent, 2: probably absent, 3: uncertain, 4: probably present, 5: definitely present, as established in previous studies. ${ }^{4,5,7}$ For images that received a score of up to 4 points on the scale, the observers only analyzed the defects qualitatively. However, when the observers scored an image as 5 (definitely present), a quantitative measurement of the defect was made using software, as depicted in Figure 2.
Image analyses were performed by the observers independently and at separate times, and the time allocated for interpretation was not limited. Both observers were blinded to the information on the sample images. Each implant was analyzed separately. All sequences of the observations were randomized and viewed randomly by both observers.

\section{Statistical analyses}

The kappa test was performed to assess intraobserver reliability and interobserver reproducibility for the detection of bone defects. Furthermore, intraclass correla- 
tion coefficients (ICCs) were calculated to determine the agreement of the linear measurements of bone defect depth performed by both observers. All CBCT-based linear measurements were compared with the measurements made using direct probes in the mandibles. The following ICC criteria were used to evaluate interobserver and intraobserver agreement: slight agreement, 0.00 to 0.20 ; fair agreement, 0.21 to 0.40 ; moderate agreement, 0.41 to 0.60 ; good agreement, 0.61 to 0.80 ; and excellent agreement, 0.81 to $1.00 .{ }^{17}$ The area under the curve (AUC), accuracy, sensitivity, and specificity were calculated using statistical software (ver. 24.0, IBM SPSS Statistics for Mac; IBM Corp., Armonk, NY, USA) at a level of significance of $5 \%$.

\section{Results}

In total, 48 TIFF images of cross-sectional CBCT slices were selected and assessed. The results of the kappa test, which was used to compare interobserver and intraobserver agreement for the detection of bone defects, are avail-

Table 3. Area under the curve values from a receiver operating characteristic curve analysis

\begin{tabular}{lcc}
\hline & $\begin{array}{c}\text { i-CAT Gendex } \\
\text { CB-500 }(120 \mathrm{kVp})\end{array}$ & $\begin{array}{c}\text { Orthopantomograph OP300 } \\
(90 \mathrm{kVp})\end{array}$ \\
\hline Observer 1 & $0.55^{*}$ & $0.59^{*}$ \\
Observer 2 & $0.50^{*}$ & $0.54 *$ \\
Mean & $0.52 \pm 0.03^{*}$ & $0.56 \pm 0.03$ \\
\hline
\end{tabular}

$* P<0.05$ able in Table 1 and showed considerable similarity. Both qualitative and quantitative measurements showed strong intraobserver and interobserver agreement, as described in Table 2. The lowest value was 0.70 and the highest value was 0.95 , with statistical significance $(P<0.05)$.

The AUC values for the observers are shown in Table 3 . The OP300 device showed a slightly higher detection rate than the CB-500 (AUC $0.56 \pm 0.03$ ), although the difference was not statistically significant. Table 4 shows the values for accuracy, sensitivity, and specificity. The highest accuracy was obtained by observer $1(75.0 \%)$, and the lowest by observer $2(70.8 \%)$. The sensitivity of both observers was the same (81.2\%). For the OP300 device, the accuracy, specificity, and sensitivity of observers 1 and 2 were equal. A comparison of AUC values using the unpaired t-test with the Welch correction is shown in Table 5 . There was no significant difference between both CBCT devices regarding the detection of defects $(P>0.05)$.

\section{Discussion}

In the present study, dental implants were placed in dry human mandibles to analyze the presence of peri-implant buccal bone defects, in accordance with previous methodologies. ${ }^{5,18}$ Of note, controversial results have been reported in studies performed on animal bones. ${ }^{4,7,11,14,15}$ Some of the aforementioned studies assessed bone defects created by chemical means, ${ }^{4,11}$ to simulate peri-implant areas of bone resorption that are commonly irregular and have undefined edges. Nevertheless, the present diagnostic study was conducted on well-delimited defects created

Table 4. Diagnostic values with confidence intervals for the detection of peri-implant defects

\begin{tabular}{|c|c|c|c|c|}
\hline & & Observer 1 & Observer 2 & $P$ value* \\
\hline \multirow[t]{3}{*}{ i-CAT Gendex CB-500 (120 kVp) } & Sensitivity & $81.2 \%(54.3-95.9)$ & $81.2 \%(54.3-95.9)$ & $P<0.05$ \\
\hline & Specificity & $62.5 \%(24.5-91.5)$ & $50.0 \%(15.7-84.3)$ & $P<0.05$ \\
\hline & Accuracy & $75.0 \%(53.3-90.2)$ & $70.8 \%(48.9-87.4)$ & $P<0.05$ \\
\hline \multirow[t]{3}{*}{ Orthopantomograph OP300 $(90 \mathrm{kVp})$} & Sensitivity & $81.2 \%(54.3-95.9)$ & $81.2 \%(54.3-95.9)$ & $P<0.05$ \\
\hline & Specificity & $62.5 \%(24.5-91.5)$ & $62.5 \%(24.5-91.5)$ & $P<0.05$ \\
\hline & Accuracy & $75.0 \%(53.3-90.2)$ & $75.0 \%(53.3-90.2)$ & $P<0.05$ \\
\hline
\end{tabular}

*P value according to the Fischer's test

Table 5. Comparison of area under the curve values using the unpaired t-test with the Welch correction

\begin{tabular}{cccc}
\hline Group & F-ratio (2.2) & $95 \%$ confidence interval & $P$ value \\
\hline i-CAT Gendex CB-500 vs. Orthopantomograph OP300 & 1.033 & $-0.21-0.28$ & $P>0.05$ \\
\hline
\end{tabular}



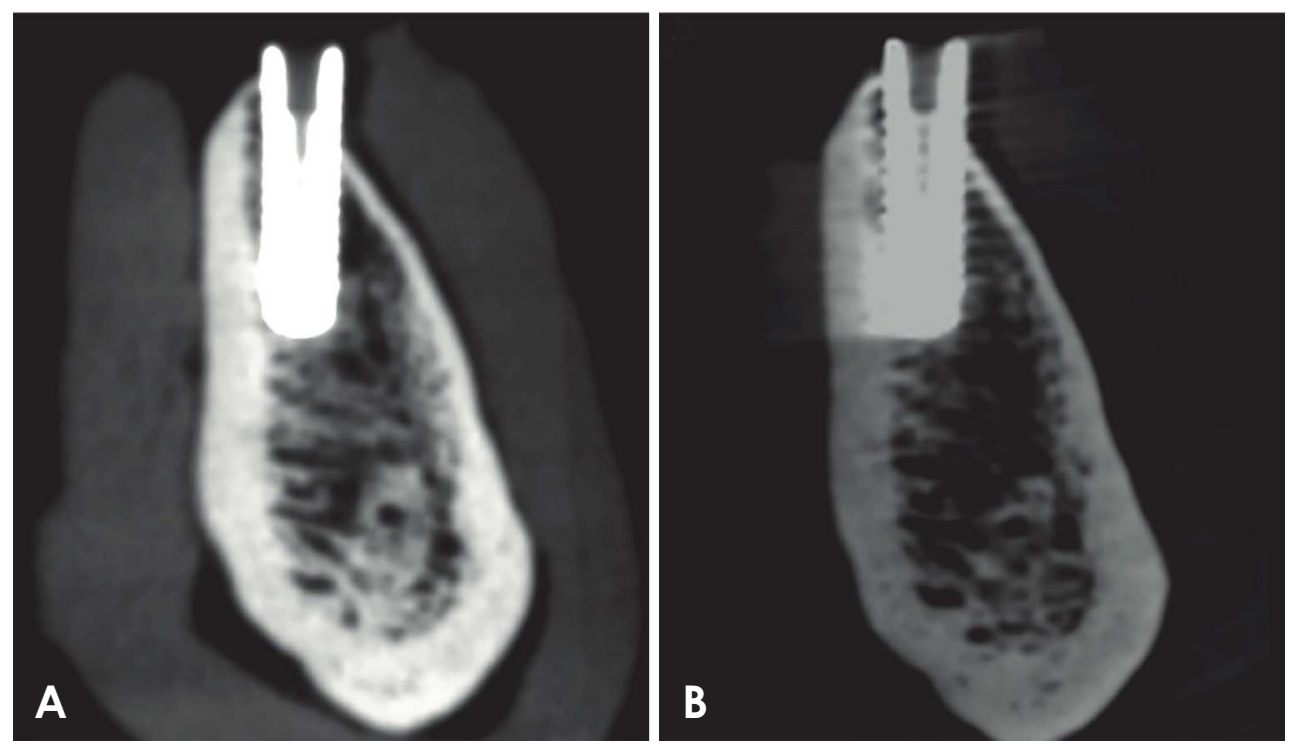

Fig. 3. Differences in image quality. A. The CB-500 images have higher contrast than the OP300 images. B. In the image obtained using the OP300 protocol, artifact formation is pronounced.

by burs with satisfactory homogeneity, enabling standardization of diagnostic performance tests, as described in previous studies. $5,7,15$

Regarding bone defect detection, the agreement values of a previous study ${ }^{19}$ ranged from 0.01 to 0.57 , whereas our results ranged from 0.61 to 0.92 . The sensitivity, specificity and AUC results shown in the present study also corroborate findings from a previous meta-analysis. ${ }^{19}$ The aforementioned authors found the following sensitivity and specificity rates for the diagnosis of CBCT defects: sensitivity, 28\%-97\%; specificity, 25\%-97\%; and AUC, 0.601.00 . Their results are partially in accordance with those found in the present study (sensitivity, $81.2 \%$; specificity, $50 \%-62.5 \%$ ), whereas the AUC values (0.51-0.75) were slightly lower, as shown in Table 4.

Schulze et al. ${ }^{12}$ summarized the theoretical constraints related to the formation of artifacts induced by titanium implants, based on simulations and geometrical considerations. The authors demonstrated the effects of varying $\mathrm{kVp}$ values, and reported that beam hardening was more prominent when there was lower energy related to $\mathrm{kVp}$. Pauwels et al. ${ }^{20}$ investigated the effects of a variety of $\mathrm{kVp}$ and current settings on image quality, as well as those of different radiation doses administered by a CBCT device (3D Accuitomo 170; J. Morita Mfg. Corp., Kyoto, Japan) comparable to those studied herein (CB-500 and OP300), and reported optimal image quality at $90 \mathrm{kVp}$. CBCT images obtained at $75 \mathrm{kVp}$ had more noise, thereby reducing defect detection. Similarly, differences in image resolution resulting from different voxel sizes and file resolutions have been described to influence linear measurements and diagnostic performance based on CBCT images. However, the difference in diagnostic performance found herein was not statistically significant, which contrasts with previous results in the literature. ${ }^{7,21}$

Unlike the results observed by Pauwels et al., ${ }^{20}$ corroborating the findings by Schulze et al., ${ }^{12}$ the OP300 device presented better specificity than the CB-500 although the OP300 acquisition protocol (with lower $\mathrm{kVp}$ settings than in the CB-500 protocol) led to the formation of more artifacts. In general, a higher number of artifacts indicates lower image quality. However, although there were more artifacts in the OP300 images than in the CB-500 images, the OP300 diagnostic values were slightly higher than those of the CB-500 (Tables 1 to 4).

Considering the values of interobserver and interobserver agreement, the results for sensitivity, specificity, and AUC could have been better if each observer had been free to manipulate the CBCT images in all 3 cross-sectional slices, instead of having to use only the sagittal image. This limitation was also reported in the study conducted by Dave et al. ${ }^{15}$ A recent study using 2 CBCT devices with different $\mathrm{kVp}$ values $(90 \mathrm{kVp} \text { and } 75 \mathrm{kVp})^{11}$ found that devices with a higher $\mathrm{kVp}$ setting yielded a higher rate of detection of peri-implant defects. In this study, it was not possible to obtain the same results with the higher $\mathrm{kVp}$ device (CB-500, $120 \mathrm{kVp}$ ); however, the results corroborate those found for the device with the same $\mathrm{kVp}$ that was evaluated in the pre- 
vious study (OP300, $90 \mathrm{kVp})$.

Researchers have observed that a higher $\mathrm{kVp}$ improves the quality of CBCT images in regard to the contrast-tonoise ratio. ${ }^{20}$ To achieve acceptable image quality with CBCT using a minimal radiation dose, current reduction is preferred over $\mathrm{kVp}$ reduction. In another study, the same authors ${ }^{15}$ showed that noise increased in protocols with the lowest current settings, thereby diminishing the quality of the CBCT image. However, the influence of current could not be evaluated in the present study, since the current acquisition protocols were too similar.

In terms merely of the quality of the images obtained using the 2 devices, the images obtained using the CB500 protocol had higher contrast than those obtained using the OP300 protocol (Fig. 3). This can be attributed to the higher $\mathrm{kVp}$ value for $\mathrm{CB}-500$ than OP300. In images with higher contrast, different tissues or structures, such as those indicated by different shades of gray, are less sharply defined and therefore more difficult to distinguish; this may have influenced the results of the diagnostic tests. The attenuation processes of the X-ray beams in contact with structures generate a composite image of different shades of gray, and artifacts from metallic materials add to the non-homogeneity of the gray values.

Within the limitations of this study, the present results suggest that the choice of CBCT devices with their respective commonly used acquisition protocols does not significantly affect diagnostic performance in detecting and measuring peri-implant bone loss.

\section{Conflicts of Interest: None}

\section{References}

1. Sanz M, Chapple IL, Working Group 4 of the VIII European Workshop on Periodontology. Clinical research on peri-implant diseases: consensus report of Working Group 4. J Clin Periodontol 2012; 39 Suppl 12: 202-6.

2. Bagis N, Kolsuz ME, Kursun S, Orhan K. Comparison of intraoral radiography and cone-beam computed tomography for the detection of periodontal defects: an in vitro study. BMC Oral Health 2015; 15: 64.

3. Kolsuz ME, Bagis N, Orhan K, Avsever H, Demiralp K. Comparison of the influence of FOV sizes and different voxel resolutions for the assessment of periodontal defects. Dentomaxillofac Radiol 2015; 44: 20150070.

4. Pinheiro LR, Scarfe WC, Augusto de Oliveira Sales M, Gaia BF, Cortes AR, Cavalcanti MG. Effect of cone-beam computed tomography field of view and acquisition frame on the detection of chemically simulated peri-implant bone loss in vitro. J Periodontol 2015; 86: 1159-65.

5. Kamburoglu K, Kolsuz E, Murat S, Eren H, Yüksel S, Paksoy
CS. Assessment of buccal marginal alveolar peri-implant and periodontal defects using a cone beam CT system with and without the application of metal artefact reduction mode. Dentomaxillofac Radiol 2013; 42: 20130176.

6. Golubovic V, Mihatovic I, Becker J, Schwarz F. Accuracy of cone-beam computed tomography to assess the configuration and extent of ligature-induced peri-implantitis defects. A pilot study. Oral Maxillofac Surg 2012; 16: 349-54.

7. de-Azevedo-Vaz SL, Vasconcelos Kde F, Neves FS, Melo SL, Campos PS, Haiter-Neto F. Detection of periimplant fenestration and dehiscence with the use of two scan modes and the smallest voxel sizes of a cone-beam computed tomography device. Oral Surg Oral Med Oral Pathol Oral Radiol 2013; 115 : 121-7.

8. de-Azevedo-Vaz SL, Alencar PN, Rovaris K, Campos PS, Haiter-Neto F. Enhancement cone beam computed tomography filters improve in vitro periimplant dehiscence detection. Oral Surg Oral Med Oral Pathol Oral Radiol 2013; 116: 6339.

9. de-Azevedo-Vaz SL, Peyneau PD, Ramirez-Sotelo LR, Vasconcelos Kde F, Campos PS, Haiter-Neto F. Efficacy of a cone beam computed tomography metal artifact reduction algorithm for the detection of peri-implant fenestrations and dehiscences. Oral Surg Oral Med Oral Pathol Oral Radiol 2016; 121: 550-6.

10. Mengel R, Kruse B, Flores-de-Jacoby L. Digital volume tomography in the diagnosis of peri-implant defects: an in vitro study on native pig mandibles. J Periodontol 2006; 77: 123441.

11. Pinheiro LR, Scarfe WC, de Oliveira Sales MA, Gaia BF, Cortes AR, Gusmão Paraiso Cavalcanti M. Effectiveness of periapical radiography versus cone beam computed tomography with different kilovoltage settings in the detection of chemically created peri-implant bone defects: an in vitro study. Int J Oral Maxillofac Implants 2017; 32: 741-50.

12. Schulze R, Heil U, Gross D, Bruellmann DD, Dranischnikow E, Schwanecke U, et al. Artefacts in CBCT: a review. Dentomaxillofac Radiol 2011; 40: 265-73.

13. Kim JH, Arita ES, Pinheiro LR, Yoshimoto M, Watanabe PC, Cortes AR. Computed tomographic artifacts in maxillofacial surgery. J Craniofac Surg 2018; 29: e78-80.

14. Ritter L, Elger MC, Rothamel D, Fienitz T, Zinser M, Schwarz $\mathrm{F}$, et al. Accuracy of peri-implant bone evaluation using cone beam CT, digital intra-oral radiographs and histology. Dentomaxillofac Radiol 2014; 43: 20130088.

15. Dave M, Davies J, Wilson R, Palmer R. A comparison of cone beam computed tomography and conventional periapical radiography at detecting peri-implant bone defects. Clin Oral Implants Res 2013; 24: 671-8.

16. Kühl S, Zürcher S, Zitzmann NU, Filippi A, Payer M, Dagassan-Berndt D. Detection of peri-implant bone defects with different radiographic techniques - a human cadaver study. Clin Oral Implants Res 2016; 27: 529-34.

17. Landis JR, Koch GG. The measurement of observer agreement for categorical data. Biometrics 1977; 33: 159-74.

18. Bohner LO, Tortamano P, Marotti J. Accuracy of linear measurements around dental implants by means of cone beam computed tomography with different exposure parameters. 
Dentomaxillofac Radiol 2017; 46: 20160377.

19. Bohner LO, Mukai E, Oderich E, Porporatti AL, Pacheco-Pereira $\mathrm{C}$, Tortamano $\mathrm{P}$, et al. Comparative analysis of imaging techniques for diagnostic accuracy of peri-implant bone defects: a meta-analysis. Oral Surg Oral Med Oral Pathol Oral Radiol 2017; 124: 432-40.e5.
20. Pauwels R, Silkosessak O, Jacobs R, Bogaerts R, Bosmans H, Panmekiate $\mathrm{S}$. A pragmatic approach to determine the optimal $\mathrm{kVp}$ in cone beam CT: balancing contrast-to-noise ratio and radiation dose. Dentomaxillofac Radiol 2014; 43: 20140059.

21. Ziefle M. Effects of display resolution on visual performance. Hum Factors 1998; 40: 554-68. 